\title{
INTRA-EYE PRESSURE RANGE AND PULSE PROFILES IN NORMALS WITH THE PULSAIR NON-CONTACT TONOMETER
}

\author{
STEPHEN A. VERNON \\ Nottingham
}

\begin{abstract}
SUMMARY
The difference in intraocular pressure (IOP) between the highest and lowest measurements on an eye with noncontact tonometry (the range) has been reported to be less than $4 \mathrm{mmHg}$ in most normals. The range of IOP to be expected with the Keeler Pulsair in normals over the age of 50 years was determined by analysing the results of IOP recordings on 741 normal individuals. Between $56 \%$ and $62 \%$ of eyes recorded a range $>4 \mathrm{mmHg}$ and between $4 \%$ and $8 \%$ a range $>10 \mathrm{mmHg}$ when four pulses per eye were used. Population pulse profile analysis indicated that this was not due to an erroneously high first pulse in a set of four, and that IOP asymmetry previously reported when screening females is due to a generalised tendency for IOPs in right eyes to measure higher than those recorded in the left. The occurrence of a range of IOP recordings up to $10 \mathrm{mmHg}$ in an eye is relatively common with the Pulsair and individuals undergoing glaucoma screening with this instrument should not be referred purely on the basis of a large IOP range.
\end{abstract}

Non-contact tonometry (NCT) samples the ocular pulse by causing a momentary applanation event on the cornea with a ramped jet of air. In order to estimate the mean intraocular pressure (IOP) in an eye, a number of NCT pulses are used. The Keeler Pulsair NCT was introduced in $1988 .{ }^{1}$ We have reported previously on the effect of altering the number of pulses used to estimate the IOP in a population at risk of glaucoma, ${ }^{2}$ and the asymmetry to be expected in normal individuals when using the Keeler Pulsair NCT. ${ }^{3}$ In the latter study we demonstrated that, when screening for glaucoma, normal individuals record an IOP which is on average $0.63 \mathrm{mmHg}$ higher in the right eye than the left eye when the right eye is tested first. The effect is marked in females, reaching a level of statistical significance of $p<0.0001$; in males asymmetry just fails to reach statistical significance $(p<0.07)$.

Correspondence to: Mr S. A. Vernon, FRCS, FCOphth, Academic Unit of Ophthalmology, University Hospital, Nottingham NG7 2UH, UK.
Two questions remain unanswered. Firstly, is the first pulse of the series of four used to calculate the IOP in an eye likely to be higher than the remaining three pulses (as a result of patient anxiety)? If so, this might explain the asymmetry as right eyes were always measured first. Secondly, what is the range of IOPs in a set of four in a normal population? Do right eyes differ from left and do males differ from females?

Piltz et al., using the American Optical NCT, reported the range of IOPs (highest minus lowest IOP recorded in a set of pulses on an eye) to be significantly increased in subjects with glaucoma. ${ }^{4}$ An IOP range greater than $4 \mathrm{mmHg}$ within a set of four NCT readings might therefore be considered to be significant when screening for glaucoma.

This paper studies the range of IOPs found in a group of 741 normal individuals when screening for glaucoma with the Pulsair NCT and examines the 'pulse profiles' from a population of individual measurements within the set of four used to calculate the IOP.

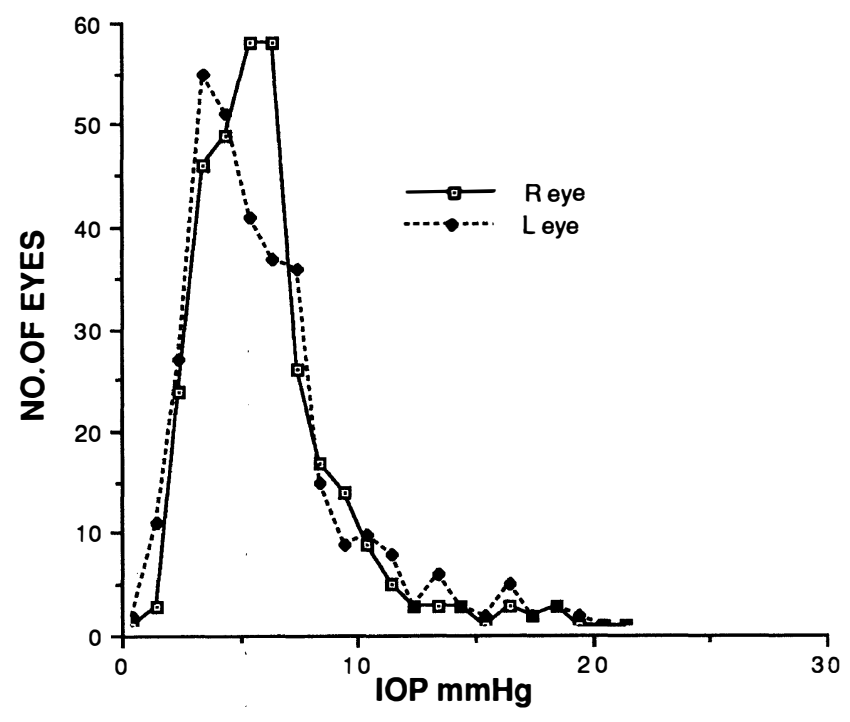

Fig. 1. Range of IOPs in a set of four: males $(\mathrm{n}=309)$. 


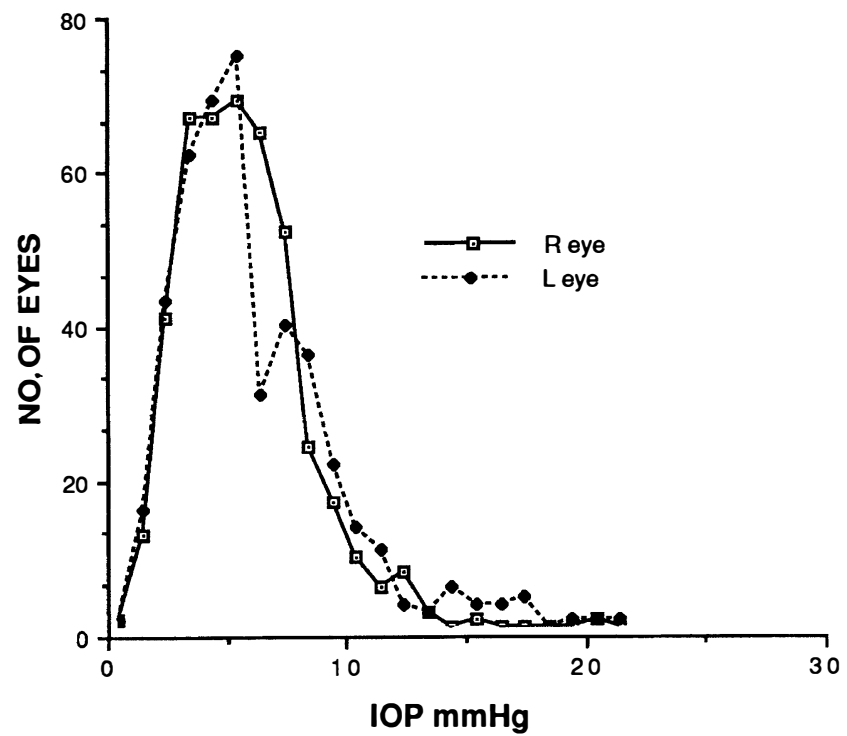

Fig. 2. Range of IOPs in a set of four: females $(\mathrm{n}=432)$.

\section{MATERIALS AND METHODS}

The study uses IOP data derived from an epidemiological project that has been the subject of a number of previous reports. ${ }^{2,3,5-7}$ Of all eligible persons aged 50 years and over from a general practice population $88.5 \%$ were screened for glaucoma using a protocol described in detail elsewhere..$^{5}$ All 874 persons had IOP assessment of both eyes by a single operator using the Pulsair NCT. IOP was recorded as the mean of four consecutive readings with the eye in the primary position; right eyes were always measured first.

A subpopulation comprising 741 of these individuals had IOPs $<22 \mathrm{mmHg}$ in both eyes, a normal Henson CFS2000 field examination, and normal optic discs as assessed by an experienced observer 'blind' to IOP and field data. This group, further examined in this study, can therefore be considered a representative sample of the normal population aged 50 years and over.

For each eye, the lowest IOP pulse of the four recorded was subtracted from the highest to produce a 'range'. In addition, pulse profiles were calculated by correlating single-pulse IOP readings in each population subset. For example, the frequencies of IOPs recorded from all first pulses from male right eyes were charted against the IOP, producing a distribution plot. This permits one pulse profile to be compared statistically and graphically with any of the seven similar profiles constructed from the eight pulses used per person (four right, four left).

\section{RESULTS}

Fig. 1 shows the range found in male right $(\mathrm{R})$ and left $(\mathrm{L})$

Table I. Mean range of IOPs ( $\mathrm{mmHg}$ ) with $95 \%$ confidence intervals of the means

\begin{tabular}{lcc}
\hline & Mean & $95 \%$ CI \\
\hline Male R eyes & 5.53 & \pm 0.31 \\
Male L eyes & 5.60 & \pm 0.62 \\
Female R eyes & 5.22 & \pm 0.49 \\
Female L eyes & 5.65 & \pm 0.53 \\
\hline
\end{tabular}

Table II. Variation of mean range of IOPs $(\mathrm{mmHg})$ with number of pulses per eye used

\begin{tabular}{lccc}
\hline & \multicolumn{2}{l}{ Range with: } & \\
\cline { 2 - 4 } & 2 pulses & 3 pulses & 4 pulses \\
\hline Male R eye & 2.90 & 4.33 & 5.53 \\
Male L eye & 3.04 & 4.53 & 5.60 \\
Female R eye & 3.01 & 4.25 & 5.22 \\
Female L eye & 3.07 & 4.71 & 5.65 \\
\hline
\end{tabular}

eyes and Fig. 2 the range for females. There is no significant difference between the distribution of IOP range in any subset. Fifty-seven per cent of female $\mathrm{R}$ and $\mathrm{L}$ eyes had a range $>4 \mathrm{mmHg} ; 62 \%$ of male $\mathrm{R}$ eyes and $56 \%$ of male $\mathrm{L}$ eyes had a range $>4 \mathrm{mmHg}$. Four per cent of female $\mathrm{R}$ and $8 \%$ of female $\mathrm{L}$ eyes had a range $>10 \mathrm{mmHg}$. Five per cent of male $\mathrm{R}$ and $8 \%$ of male $\mathrm{L}$ eyes had a range $>10 \mathrm{mmHg}$.

Table I indicates the mean range to be found in the population subsets and the $95 \%$ confidence intervals of the means. Table II indicates how the range would decrease if fewer pulses were used per eye (' 2 pulses' is based on the first two recorded, etc).

Fig. 3 shows the four pulse profiles from male R eyes. Each of the eight profiles in both males and females approximated to a normal distribution and therefore a twotailed $t$-test was used to compare one profile with any of the others from that sex. There are therefore 28 possible comparisons that can be made in males and 28 in females (e.g. R 1st vs. L 2nd, R 4th vs. L 3rd).

Of the 28 profile comparisons in females, significant differences were recorded in 16 , whereas in males only $3 / 28$ were statistically different $(p<0.05$ by chi-squared using Yates' correction for small groups). Profiles which are statistically different are itemised with their level of significance in Table III.

Fig. 4 demonstrates graphically the difference between the $\mathrm{R} 1$ st pulse and L 2 nd pulse profiles in females, and

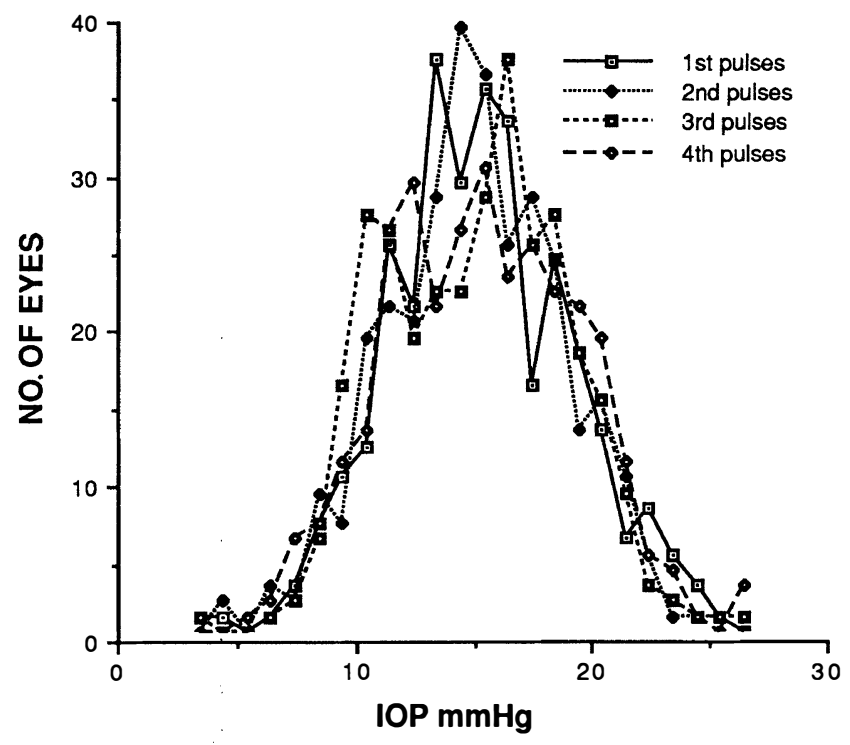

Fig. 3. Pulse profiles of male right eyes. No profile is significantly different from any other. 
Table III. Pulse profile comparisons. Only those values significant using the two-tailed $t$-test are shown

\begin{tabular}{lc}
\hline Profile comparison & Significance level $(p)$ \\
\hline FR $1>$ FR2 & $<0.05$ \\
FR $1>$ FL1 & $<0.0001$ \\
FR $1>$ FL2 & $<0.0001$ \\
FR $1>$ FL3 & $<0.003$ \\
FR $1>$ FL4 & $<0.0001$ \\
FR2 $>$ FL1 & $<0.002$ \\
FR2 $>$ FL2 & $<0.001$ \\
FR2 $>$ FL4 & $<0.02$ \\
FR3 $>$ FL1 & $<0.0002$ \\
FR3 $>$ FL2 & $<0.0001$ \\
FR3 $>$ FL4 & $<0.002$ \\
FR4 $>$ FL1 & $<0.0005$ \\
FR4 $>$ FL2 & $<0.0003$ \\
FR 4 FL4 & $<0.005$ \\
FR3 $>$ FL1 & $<0.05$ \\
FR3 $>$ FL2 & $<0.04$ \\
MR $1>$ ML2 & $<0.03$ \\
MR4 > ML1 & $<0.02$ \\
MR4 $>$ ML2 & $<0.007$ \\
\hline
\end{tabular}

F, female; M, male; L, teft; R, right.

shows a shift to the right in the normal distribution. This is typical of instances when a right pulse profile is different from a left. In females, $1 \mathrm{i} / 14 \mathrm{R}$ versus $\mathrm{L}$ profile comparisons showed this effect. This indicates a generalised tendency for all pulses measured in $\mathrm{R}$ eyes to be higher than those measured in $L$ eyes. In males only $3 / 14 R$ versus $L$ profile comparisons were significantly different, indicating a much decreased tendency for IOPs in R eyes to record higher than those from $\mathrm{L}$ eyes.

Not all significant differences in females were between right and left profiles. Female R 1st is different from female R 2nd (see Fig. 5), but the lower IOP frequencies are similar, a change in the mid-range/upper frequencies being sufficient to induce statistical significance at the $p<0.05$ level. (Although $\mathrm{R} 1 \mathrm{st}$ is statistically greater than $R$ 2nd, note that $R$ 2nd is still greater than $L$ 1st, $L$ 2nd and L 4th.) A similar profile curve explained why L 3rd pulse was greater than $\mathrm{L} 2 \mathrm{nd}$ pulse and therefore why

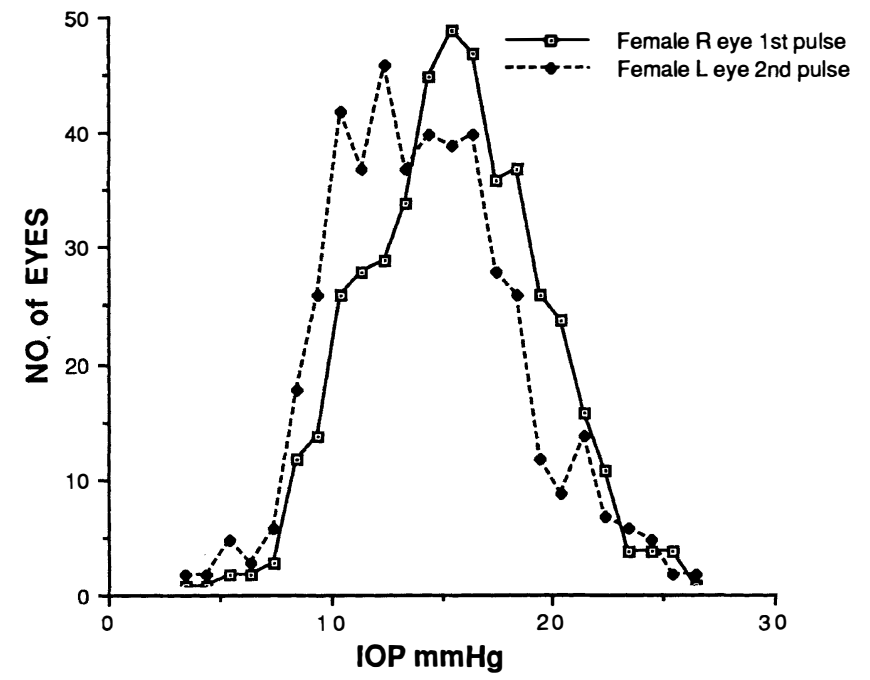

Fig. 4. Pulse profile: female right 1 st (FRI) versus female left 2nd (FR2). FRI $>$ FL2 $(\mathrm{p}<0.0001)$.

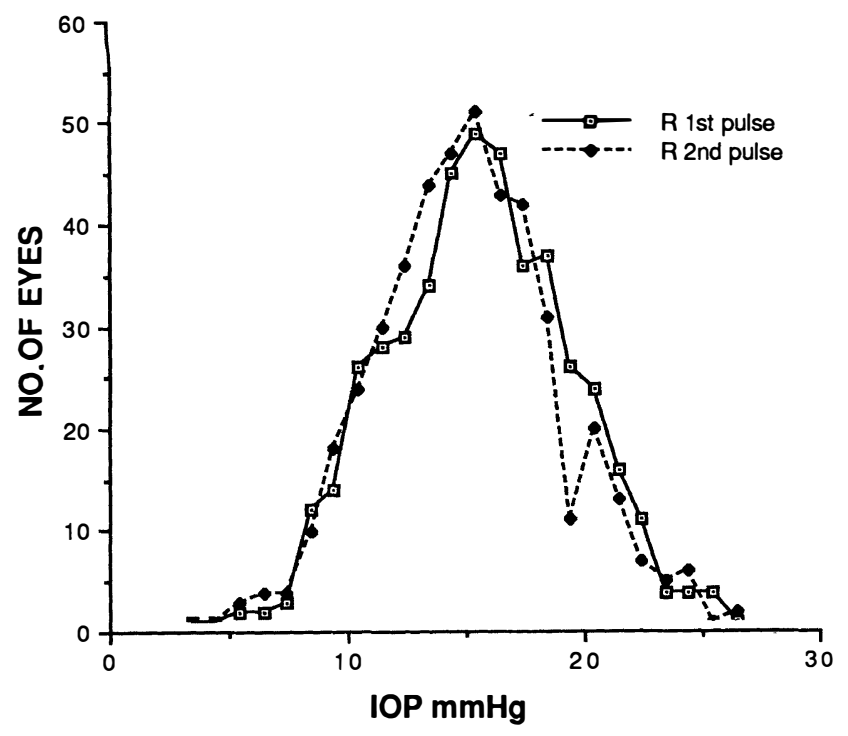

Fig. 5. Pulse profile: female right 1 st (FR1) versus female right 2 nd $(F R 2)$. FRI $>F R 2(\mathrm{p}<0.05)$.

L 3rd was not significantly different from R 2nd, R 3rd and R 4th.

\section{DISCUSSION}

The range of IOPs found using the forerunner to the Pulsair, the American Optical NCT, has been reported to be less than $4 \mathrm{mmHg}$ in almost $90 \%$ of normals. ${ }^{8}$ On repeated applanation in 18 normal eyes of 10 patients involving 144 sets of four pulses, Piltz found that a range of $>4 \mathrm{mmHg}$ in a set of four was found in only $12.5 \%$ of sets. No mention of the age or sex of the patients was made and as each eye had eight sets of readings, individuals would be considerably experienced in NCT as data collection proceeded.

Armstrong has reported a range $>10 \mathrm{mmHg}$ to occur in $32 \%$ of cases when using the Pulsair, but again no details of age or sex were given in this paper. ${ }^{9}$ Our study, on a much larger population, indicates that in a screening programme with a single operator performing Pulsair NCT between $56 \%$ and $62 \%$ of normal eyes will have a range of $>4 \mathrm{mmHg}$, and between $4 \%$ and $8 \%>10 \mathrm{mmHg}$ when four pulses per eye are used.

The mean amplitude of the intraocular pulse in normals has been reported to be between 1.8 and $2.8 \mathrm{mmHg} .^{10,11}$ The mean range of around $5.5 \mathrm{mmHg}$ found in this study using four pulses per eye further emphasises the importance of taking a series of measurements with the Pulsair in order to estimate the true $\mathrm{IOP}^{2}$ It also suggests that individual pulses may record relatively inaccurate IOPs. Although the manufacturers advise taking the mean of all readings, from Figs. 1 and 2 we deduce that it is probably wise to repeat a set of four where the range exceeds $8 \mathrm{mmHg}$.

Although there was not a significant difference between the eyes of either sex with respect to the range of IOPs recorded, we have demonstrated that any of the pulses from female right eyes are likely to be higher than those 
from the left. It should be emphasised that very few of the subjects in this study had had Pulsair NCT on a previous occasion and that right eyes were always tested first. If anxiety is the primary reason for Pulsair NCT asymmetry, ${ }^{3}$ it is clear that this is much more of a factor in females.

It would appear, therefore, that the Pulsair, which uses a lower pulse pressure and volume than the American Optical instrument (personal communication from Keeler Ltd.), can be expected to record higher ranges of pressures within a set of four in normal subjects. However, it remains to be seen whether asymmetry and relatively large ranges persist when subjects have prior experience of the instrument. The finding of a large range of IOPs within a set should not, therefore, be used as a criterion for referral if the mean is still acceptable and disc and fields appear normal. Individuals recording a range $>8 \mathrm{mmHg}$ should have all four readings repeated.

The author acknowledges the support of the International Glaucoma Association and Merck Sharp and Dohme Ltd in the epidemiological study. The Keeler Pulsair was loaned by the company, in which the author has no financial interest.

Key words: Glaucoma, IOP range, Non-contact tonometry, Normals, Screening.

\section{REFERENCES}

1. Fisher JH, Watson PG, Spaeth G. A new handheld tonometer. Eye 1988;2:238-42.

2. Vernon SA, Jones SJ, Henry DJ. Maximising the sensitivity and specificity of non-contact tonometry in glaucoma screening. Eye 1991;5:491-3.

3. Vernon SA, Jones SJ. Intraocular pressure asymmetry in a population tested with the Pulsair noncontact tonometer. Eye 1991;5:674-7.

4. Piltz JR, Starita R, Miron M, Henkind P. Momentary fluctuations in intraocular pressure in normal and glaucomatous eyes. Am J Ophthalmol 1985;99:333-9.

5. Vernon SA, Henry DJ, Cater L, Jones SJ. Screening for glaucoma in the community by non-ophthalmologically trained staff using semiautomated equipment. Eye 1990;4:89-97.

6. Jones SJ, Vernon SA, Cater L, Henry DJ. Costing a community based screening programme for the detection of glaucoma. Eye 1990;4:98-102.

7. Vernon SA, Henry DJ, Jones SJ. Calculating the predictive power of the Henson field screener in a population at risk of glaucomatous field loss. Br J Ophthalmol 1990;74:220-2.

8. Forbes M, Pico G, Grolman B. A noncontact applanation tonometer. Arch Ophthalmol 1974;91:134-40.

9. Armstrong TA. Evaluation of the Tono-pen and the Pulsair tonometers. Am J Ophthalmol 1990;109:716-20.

10. Trew DR, Smith SE. Postural studies in pulsatile ocular blood flow. I. Ocular hypertension and normotension. Br J Ophthalmol 1991;75:66-70.

11. Perkins ES. The ocular pulse. Curr Eye Res 1981;1:19-23. 